\title{
The Formalization of The Arithmetic System on The Ground of The Atomic Logic
}

\author{
T. J. Stepień, L. T. Stepień \\ The Pedagogical University of Cracow, ul. Podchorazych 2, 30 - 084 Krakow, Poland.
}

Received: May 21, 2015 / Accepted: June19, 2015 / Published: September 25, 2015.

\begin{abstract}
This paper is a continuation of the paper [16]. Namely, in [16] we have introduced, among others, the definition of the atomic entailment and we have constructed the system $\stackrel{\Pi}{S}$, which is based on the atomic entailment. In this paper we show that the classical Arithmetic can be based on the Atomic Logic (see [17]).
\end{abstract}

Keywords: Atomic entailment, Arithmetic System, Peano's Arithmetic System, classical Arithmetic

\section{Notational Preliminaries}

We assume that $\rightarrow, \sim, \vee, \wedge, \equiv$ denote the connectives of implication, negation, disjunction, conjunction and equivalence, respectively. We use $\Rightarrow, \neg, \Leftrightarrow, \&, \mathbb{V}, \forall, \exists$ as metalogical symbols. Next,

$$
A t_{0}=\left\{p, p_{1}, p_{2}, \ldots, q, q_{1}, q_{2}, \ldots, s, s_{1}, s_{2}, \ldots t, \ldots\right\}
$$

denotes the set of all propositional variables. $S_{0}$ is the set of all well-formed formulas, which are built in the usual manner from propositional variables and by means of logical connectives. $P_{0}(\phi)$ denotes the set of all propositional variables occuring in $\phi\left(\phi \in S_{0}\right)$. $R_{S_{0}}$ denotes the set of all rules over $S_{0}$. Hence, for every $r \in R_{S_{0}},\langle\Pi, \phi\rangle \in r$, where $\Pi \subseteq S_{0}$ and $\phi \in S_{0}$ and $\Pi$ is a set of premisses and $\phi$ is a conclusion. Hence, $r_{*}^{0}$ denotes here the rule of simultaneous substitution for propositional variables. $\langle\{\phi\}, \psi\rangle \in$ $r_{*}^{0} \Leftrightarrow\left[h^{e}(\phi)=\psi\right]$, where $h^{e}$ is the extension of the mapping $e: A t_{0} \rightarrow S_{0}\left(e \in \varepsilon_{*}^{0}\right)$ to endomorphism $h^{e}: S_{0} \rightarrow S_{0}$, where

1. $h^{e}(\phi)=e(\phi)$, for $\phi \in A t_{0}$

2. $h^{e}(\sim \phi)=\sim h^{e}(\phi)$

3. $h^{e}(\phi F \psi)=h^{e}(\phi) F h^{e}(\psi)$, for $F \in\{\rightarrow, \vee, \wedge, \equiv\}$ and for every $\phi, \psi \in S_{0}$.

Corresponding author: L. T. Stepień, The Pedagogical University of Cracow, Kraków, Poland. E-mail: sfstepie@cyf-kr.edu.pl, http://www.ltstepien.up.krakow.pl.
Thus, $\varepsilon_{*}^{0}$ is a class of functions $e: A t_{0} \rightarrow S_{0}$ (for details, see [5], cf. [2]). $r_{0}^{0}$ denotes here the Modus Ponens rule in propositional calculus. $R_{0 *}=\left\{r_{0}^{0}, r_{*}^{0}\right\}$ (for details, see [2], [5]). A logical matrix is a pair $\mathfrak{M}=\left\{U, U^{\prime}\right\}, U$ is an abstract algebra and $U^{\prime}$ is a subset of the universe $U$, i.e. $U^{\prime} \subseteq U$. Any $a \in U^{\prime}$ is called a distinguished element of the matrix $\mathfrak{M} . E(\mathfrak{M})$ is the set of all formulas valid in the matrix $\mathfrak{M} . \mathfrak{M}_{2}$ denotes the classical two-valued matrix.

Hence, $Z_{2}$ is the set of all formulas valid in the classical matrix $\mathfrak{M}_{2}$ (see [2], [5]).

The symbols $x_{1}, x_{2}, \ldots$ are individual variables. $a_{1}, a_{2}, \ldots$ are individual constants. $V$ is the set of all individual variables. $C$ is the set of all individual constants. $\quad P_{i}^{n}(i, n \in \mathcal{N}=\{1,2, \ldots\}) \quad$ are $n$-ary predicate letters. The symbols $f_{i}^{n}(i, n \in \mathcal{N})$ are $n$-ary function letters. The symbols $\wedge x_{k}, \vee x_{k}$ are quantifiers. $\Lambda x_{k}$ is the universal quantifier and $\vee x_{k}$ is the existential quantifier. The function letters, applied to the individual variables and individual constants, generate terms. The symbols $t_{1}, t_{2}, \ldots$ are terms. $T$ is the set of all terms. $V \cup C \subseteq T$.

The predicate letters, applied to terms, yield simple formulas, i.e. if $P_{i}^{k}$ is a predicate letter and $t_{1}, \ldots, t_{k}$ are terms, then $P_{i}^{k}\left(t_{1}, \ldots, t_{k}\right)$ is a simple formula. 
Smp is the set of all simple formulas. Next, $A t_{1}$ is the set of all atomic formulas, where $A t_{1}=$ $\left\{P_{i}^{k}\left(x_{j_{1}}, \ldots, x_{j_{k}}\right): k, i, j_{1}, \ldots, j_{k} \in \mathcal{N}\right\}$. At last, $S_{1}$ is the set of all well-formed formulas. $F V(\phi)$ denotes the set of all free variables occuring in $\phi$, where $\phi \in S_{1}$. $x_{k} \in F f\left(t_{m}, \phi\right)$ expresses that $x_{k}$ is free for term $t_{m}$ in $\phi$. By $x_{k} / t_{m}$ we denote the substitution of the term $t_{m}$ for the individual variable $x_{k} . P_{1}(\phi)$ denotes the set of all predicate letters occuring in $\phi\left(\phi \in S_{1}\right)$. If $F V(\phi)=\left\{x_{1}, \ldots, x_{k}\right\}$, then $\wedge \phi=$ $\wedge x_{1} \ldots \wedge x_{k} \phi$.

$R_{S_{1}}$ denotes the set of all rules over $S_{1}$. Hence, for every $r \in R_{S_{1}}, \quad\langle\Pi, \phi\rangle \in r$, where $\Pi \subseteq S_{1}$ and $\phi \in S_{1}$ and $\Pi$ is a set of premisses and $\phi$ is a conclusion. Hence, $r_{*}^{1}$ denotes here the rule of simultaneous substitution for predicate letters. $\langle\{\phi\}, \psi\rangle \in r_{*}^{1} \Leftrightarrow\left[h^{e}(\phi)=\psi\right]$, where $h^{e}$ is the extension of the mapping $e: \operatorname{Smp} \rightarrow S_{1}\left(e \in \varepsilon_{*}^{1}\right)$ to endomorphism $h^{e}: S_{1} \rightarrow S_{1}$, where

1. $h^{e}(\phi)=e(\phi)$, for $\phi \in S m p$

2. $h^{e}(\sim \phi)=\sim h^{e}(\phi)$

3. $h^{e}(\phi F \psi)=h^{e}(\phi) F h^{e}(\psi)$,

for $F \in\{\rightarrow, \vee, \wedge, \equiv\}$

4. $h^{e}\left(\wedge x_{k} \phi\right)=\wedge x_{k} h^{e}(\phi)$

5. $h^{e}\left(\bigvee x_{k} \phi\right)=\bigvee x_{k} h^{e}(\phi)$ for every

$\phi, \psi \in S_{1}$ and $k \in \mathcal{N}$ (for details, see [6], [7]).

Next, $r_{0}^{1}$ denotes the Modus Ponens rule in predicate calculus, $r_{+}^{1}$ denotes the generalization rule. $R_{0+}=\left\{r_{0}^{1}, r_{+}^{1}\right\}, R_{0 *+}=\left\{r_{0}^{1}, r_{*}^{1}, r_{+}^{1}\right\}$. We write $X \subset Y$, if $X \subseteq Y$ and $X \neq Y$.

We assume here that for every $\alpha \in S_{1}$, if $F V(\alpha)=\left\{x_{1}, \ldots, x_{n}\right\}$, then $\alpha^{*}=\bigvee x_{1} \ldots \bigvee x_{n} \sim \alpha$. Hence, for every $\alpha \in S_{1}$, if $F V(\alpha)=\emptyset$, then $\alpha^{*}=\sim \alpha$. Analogically, for every $\alpha \in S_{0}, \alpha^{*}=\sim \alpha$.

Finally, for any $X \subseteq S_{i}$ and $R \subseteq R_{S_{i}}, C n_{i}(R, X)$ is the smallest subset of $S_{i}$, containing $X$ and closed under the rules $R \subseteq R_{S_{i}}$, where $i \in\{0,1\}$. The couple $\langle R, X\rangle$ is called a system, whenever $R \subseteq R_{S_{i}}$ and $X \subseteq S_{i}$ and $i \in\{0,1\}$. Syst $\cap A_{0}$ denotes here the class of all systems $\langle R, X\rangle$, which are based on an atomic entailment, where $R \subseteq R_{S_{0}}$ and $X \subseteq S_{0}$.
Syst $\cap A_{1}$ denotes here the class of all systems $\langle R, X\rangle$, which are based on an atomic entailment, where $R \subseteq R_{S_{1}}$ and $X \subseteq S_{1}$. Syst $\cap C_{1}$ denotes here the class of all systems $\langle R, X\rangle$, which are based on a classical entailment, where $R \subseteq R_{S_{1}}$ and $X \subseteq S_{1}$. $\phi \mid \frac{A_{0}}{R, X} \psi$ denotes that $\psi$ results atomically from $\phi$, on the ground of the system $\langle R, X\rangle$, where $R \subseteq R_{S_{0}}$ and $X \subseteq S_{0}$. Next, $\phi \mid \frac{A_{1}}{R, X} \psi$ denotes that $\psi$ results atomically from $\phi$, on the ground of the system $\langle R, X\rangle$, where $R \subseteq R_{S_{1}}$ and $X \subseteq S_{1}$. At last, $\phi \mid \frac{C_{1}}{R, X} \psi$ denotes that $\psi$ results classically from $\phi$, on the ground of the system $\langle R, X\rangle$, where $R \subseteq R_{S_{1}}$ and $X \subseteq S_{1}$ (see [16]).

Definition 1.1. The function $j: S_{1} \rightarrow S_{0}$, is

defined, as follows (see [16]):

(1) $j\left(P_{k}^{n}\left(t_{1}, \ldots, t_{n}\right)\right)=p_{k}\left(p_{k} \in A t_{0}\right)$

(2) $j(\sim \phi)=\sim j(\phi)$

(3) $j(\phi F \psi)=j(\phi) F j(\psi)$, for $F \in\{\rightarrow, \vee, \wedge, \equiv\}$

(4) $j\left(\wedge x_{k} \phi\right)=j\left(\bigvee x_{n} \phi\right)=j(\phi)$.

\section{Classical Entailment}

Definition 2.1. Let $C n_{1}(R, X)=L \neq \varnothing$ and $\phi, \psi \in S_{1}$. Then $\phi \mid \frac{C_{1}}{R, X} \psi$ iff the following conditions are satisfied, [14], [16]:

(1) $\left(\forall e \in \varepsilon_{*}^{1}\right)\left[h^{e}(\bigwedge \phi) \in L \Rightarrow h^{e}(\psi) \in L\right]$

(2) $\left(\forall e \in \varepsilon_{*}^{1}\right)\left[h^{e}\left(\left(\psi^{*} \rightarrow \phi^{*}\right) \rightarrow \phi^{*}\right) \in L \Rightarrow\right.$ $\left.h^{e}\left(\phi^{*}\right) \in L\right]$.

Definition 2.2. $\langle R, X\rangle \in$ Syst $\cap C_{1}$ iff the following condition is satisfied, [14], [16]:

$\left(\forall \phi, \psi \in S_{1}\right)\left[\wedge \phi \rightarrow \psi \in C n_{1}(R, X) \Leftrightarrow \phi \mid \frac{C_{1}}{R, X} \psi\right]$.

\section{The Classical Logic}

Let $L_{2}$ denote the set of all formulas valid in the classical predicate calculus.

Thus, (cf. [6] pp. 68 - 74):

Theorem 3.1. $C n_{1}\left(R_{0 *+}, L_{2}\right)=L_{2}$. 


\section{Atomic Entailment}

In [14], [15] and [16], we have introduced the following definitions (cf. [12], [13]):

Definition 4.1. Let $C n_{0}(R, X)=L \neq \emptyset$ and $\phi, \psi \in S_{0}$. Then $\phi \mid \frac{A_{0}}{R, X} \psi$ iff the following conditions are satisfied:

(1) $\left(\forall e \in \varepsilon_{*}^{0}\right)\left[h^{e}(\phi) \in L \Rightarrow h^{e}(\psi) \in L\right.$ $\left.\& P_{0}\left(h^{e}(\phi)\right) \subseteq P_{0}\left(h^{e}(\psi)\right)\right]$

(2) $\left(\forall e \in \varepsilon_{*}^{0}\right)\left[h^{e}\left(\left(\psi^{*} \rightarrow \phi^{*}\right) \rightarrow \phi^{*}\right) \in L \Rightarrow\right.$ $\left.h^{e}\left(\phi^{*}\right) \in L \& P_{0}\left(h^{e}\left(\psi^{*}\right)\right) \subseteq P_{0}\left(h^{e}\left(\phi^{*}\right)\right)\right]$.

Definition 4.2. $\langle R, X\rangle \in$ Syst $\cap A_{0}$ iff the following condition is satisfied:

$\left(\forall \phi, \psi \in S_{0}\right)\left[\phi \rightarrow \psi \in C n_{0}(R, X) \Leftrightarrow \phi \mid \frac{A_{0}}{R, X} \psi\right]$.

Definition 4.3. Let $C n_{1}(R, X)=L \neq \varnothing$ and $\phi, \psi \in S_{1}$. Then $\phi \mid \frac{A_{1}}{R, X} \psi$ iff the following conditions are satisfied:

(1) $\left(\forall e \in \varepsilon_{*}^{1}\right)\left[h^{e}(\Lambda \phi) \in L \Rightarrow h^{e}(\psi) \in L\right.$ $\left.\& P_{1}\left(h^{e}(\Lambda \phi)\right) \subseteq P_{1}\left(h^{e}(\psi)\right)\right]$

(2) $\left(\forall e \in \varepsilon_{*}^{1}\right)\left[h^{e}\left(\left(\psi^{*} \rightarrow \phi^{*}\right) \rightarrow \phi^{*}\right) \in L \Rightarrow\right.$ $\left.h^{e}\left(\phi^{*}\right) \in L \quad \& P_{1}\left(h^{e}\left(\psi^{*}\right)\right) \subseteq P_{1}\left(h^{e}\left(\phi^{*}\right)\right)\right]$.

Definition 4.4. $\langle R, X\rangle \in$ Syst $\cap A_{1}$ iff the following condition is satisfied:

$\left(\forall \phi, \psi \in S_{1}\right)\left[\wedge \phi \rightarrow \psi \in C n_{1}(R, X) \Leftrightarrow \phi \mid \frac{A_{1}}{R, X} \psi\right]$.

\section{The Atomic Logic}

Let us take the matrix (see [16]) $\mathfrak{M}_{D}=\left\langle\{0,1,2\},\{1,2\}, f_{D} \overrightarrow{ }, f_{D} \equiv, f_{D}^{\vee}, f_{D}^{\wedge}, f_{D}^{\sim}\right\rangle$, where:

\begin{tabular}{c|c|c|c}
$f_{D}$ & 0 & 1 & 2 \\
\hline 0 & 1 & 1 & 1 \\
\hline 1 & 0 & 1 & 0 \\
\hline 2 & 0 & 1 & 2
\end{tabular}

\begin{tabular}{c|c|c|c}
$f_{D}^{\equiv}$ & 0 & 1 & 2 \\
\hline 0 & 1 & 0 & 0 \\
\hline 1 & 0 & 1 & 0 \\
\hline 2 & 0 & 0 & 2
\end{tabular}

\begin{tabular}{c|c|c|c}
$f_{D}^{\vee}$ & 0 & 1 & 2 \\
\hline 0 & 0 & 1 & 0 \\
\hline 1 & 1 & 1 & 1 \\
\hline 2 & 0 & 1 & 2
\end{tabular}

\begin{tabular}{c|c|c|c}
$f_{D}^{\wedge}$ & 0 & 1 & 2 \\
\hline 0 & 0 & 0 & 0 \\
\hline 1 & 0 & 1 & 1 \\
\hline 2 & 0 & 1 & 2
\end{tabular}

\begin{tabular}{c|c}
$f_{\tilde{D}}^{\sim}$ & \\
\hline 0 & 1 \\
\hline 1 & 0 \\
\hline 2 & 2
\end{tabular}

It should be noticed here that the matrix $\mathfrak{M}_{D}^{\prime}=$ $\left\langle\{0,1,2\},\{1,2\}, f_{D}, f_{D}^{\sim}\right\rangle$ was investigated by

B. Sobocinski (see [9], [10]).

Next, we define $T_{D}$, putting:

Definition 5.1. $T_{D}=E\left(\mathfrak{M}_{D}\right)$.

In [10] (see [11]) we have proved the following:

Theorem 5.2. The system $\left\langle R_{0 *}, T_{D}\right\rangle$ is

axiomatizable.

Theorem 5.3. Let $\phi, \psi \in S_{0}$ and $\left(\exists e \in \varepsilon_{*}^{0}\right)\left[h^{e}(\phi) \in T_{D}\right]$.

Then $\phi \rightarrow \psi \in \mathrm{Cn}_{0}\left(R_{0 *}, T_{D}\right)$ iff

$$
\begin{gathered}
\left(\forall e \in \varepsilon_{*}^{0}\right)\left[h^{e}(\phi) \in T_{D} \Rightarrow h^{e}(\psi) \in T_{D}\right. \\
\left.\& P_{0}\left(h^{e}(\phi)\right) \subseteq P_{0}\left(h^{e}(\psi)\right)\right] .
\end{gathered}
$$

Now we define the system $\hat{S}$ of the Atomic Logic, as follows:

Definition 5.4. $\hat{S}=\left\langle R_{0}, T_{D}\right\rangle$.

Next, we define the set $L_{D}$, putting (see [16]):

Definition 5.5. $L_{D}=\left\{\phi \in L_{2}: j(\phi) \in T_{D}\right\}$.

The system $\stackrel{\Pi}{S}$ of the Atomic Logic,

is defined, as follows (see [16]):

Definition 5.6. $\stackrel{\Pi}{S}=\left\langle R_{0+}, L_{D}\right\rangle$.

\section{The Fundamental Properties of the Systems}

$\left\langle R_{0}, T_{D}\right\rangle,\left\langle R_{0+}, L_{D}\right\rangle$ and $\left\langle R_{0+}, L_{2}\right\rangle$

In [16] we have proved the following:

Theorem 6.1. $\left\langle R_{0}, T_{D}\right\rangle \in$ Syst $\cap A_{0}$.

Theorem 6.2. $\left\langle R_{0+}, L_{D}\right\rangle \in$ Syst $\cap A_{1}$.

Theorem 6.3. $\left\langle R_{0+}, L_{2}\right\rangle \in$ Syst $\cap C_{1}$. 


\section{The Main Result}

\section{Arithmetic terminology.}

Let $S_{A}$ denote the set of all well-formed formulas of the Arithmetic System. $F V_{A}(\phi)$ denotes the set of all free variables occuring in $\phi$, where $\phi \in S_{A}$. Hence, $\bar{S}_{A}=\left\{\phi \in S_{A}: F V_{A}(\phi)=\emptyset\right\}$. $\operatorname{Pr}(\phi)$ denotes the set of all predicate letters occuring in $\phi$, where $\phi \in S_{A} . R_{S_{A}}$ denotes the set of all rules over $S_{A}$. For any $X \subseteq S_{A}$ and for any $R \subseteq R_{S_{A}}, \operatorname{Cn}(R, X)$ is the smallest subset of $S_{A}$, containing $X$ and closed under the rules of $R$. The couple $\langle R, X\rangle$ is called a system, whenever $R \subseteq R_{S_{A}}$ and $X \subseteq S_{A} . R_{0+}^{P}=\left\{r_{0}^{P}, r_{+}^{P}\right\}$, where $\left\{r_{0}^{P}, r_{+}^{P}\right\} \subseteq R_{S_{A}} . r_{0}^{P}, r_{+}^{P}$ are Modus Ponens and generalization rule in the Arithmetic System, respectively. Next (cf. [3], [4], [8]):

(1) $\psi^{1}: \wedge x_{1} x_{1}=x_{1}$

(2) $\psi^{2}: \wedge x_{1} \wedge x_{2}\left(x_{1}=x_{2} \rightarrow x_{2}=x_{1}\right)$

(3) $\psi^{3}: \wedge x_{1} \wedge x_{2} \wedge x_{3}\left(x_{1}=x_{2} \rightarrow\left(x_{2}=x_{3} \rightarrow\right.\right.$ $\left.\left.x_{1}=x_{3}\right)\right)$

(4) $\psi^{4}: \wedge x_{1} \wedge x_{2} \wedge x_{3} \wedge x_{4}\left(x_{1}=x_{2} \rightarrow\left(x_{3}=x_{4} \rightarrow\right.\right.$ $\left.\left.\left(x_{1}+x_{3}=x_{2}+x_{4}\right)\right)\right)$

(5) $\psi^{5}: \wedge x_{1} \wedge x_{2} \wedge x_{3} \wedge x_{4}\left(x_{1}=x_{2} \rightarrow\left(x_{3}=x_{4} \rightarrow\right.\right.$ $\left.\left.\left(x_{1} \cdot x_{3}=x_{2} \cdot x_{4}\right)\right)\right)$

(6) $\psi^{6}: \wedge x_{1} \wedge x_{2} \wedge x_{3} \wedge x_{4}\left(x_{1}=x_{2} \rightarrow\left(x_{3}=x_{4} \rightarrow\right.\right.$ $\left.\left.\left(x_{1}<x_{3} \rightarrow x_{2}<x_{4}\right)\right)\right)$

(7) $\psi^{7}: \wedge x_{1} \sim\left(1=x_{1}+1\right)$

(8) $\psi^{8}: \wedge x_{1} \wedge x_{2}\left(x_{1}+1=x_{2}+1 \rightarrow x_{1}=x_{2}\right)$

(9) $\psi^{9}: \wedge x_{1} \wedge x_{2}\left(x_{1}+\left(x_{2}+1\right)=\left(x_{1}+x_{2}\right)+1\right)$

(10) $\psi^{10}: \wedge x_{1}\left(x_{1} \cdot 1=x_{1}\right)$

(11) $\psi^{11}: \wedge x_{1} \wedge x_{2}\left[x_{1} \cdot\left(x_{2}+1\right)=\left(x_{1} \cdot x_{2}\right)+x_{1}\right]$

(12) $\psi^{12}: \wedge x_{1} \wedge x_{2}\left[x_{1}<x_{2} \equiv \vee x_{3}\left(x_{1}+x_{3}=x_{2}\right)\right]$

(13) $\psi^{13}:\left(A(1) \wedge \wedge x_{1}\left(A\left(x_{1}\right) \rightarrow A\left(x_{1}+1\right)\right)\right) \rightarrow$

$\wedge x_{1} A\left(x_{1}\right)$,

where $A(1), A\left(x_{1}\right), A\left(x_{1}+1\right) \in S_{A}$.

Next, $X_{P}=\left\{\psi^{1}, \psi^{2}, \psi^{3}, \psi^{4}, \psi^{5}, \psi^{6}, \psi^{7}, \psi^{8}, \psi^{9}, \psi^{10}\right.$, $\left.\psi^{11}, \psi^{12}\right\}$.

$Y_{P}$ denotes here the set of all axioms of induction. At last, $L_{2}^{r}$ and $X_{r}$ denote the set of all logical axioms of the Arithmetic System and the set of all specific axioms of the Arithmetic System, where
$L_{2}^{r}, X_{r} \subseteq S_{A}$, respectively. $S x$ denotes here the successor of $x$ (see [1]).

$\psi^{14}$ denotes the formula (see [3])

$\wedge x_{1} \wedge x_{2}\left[\bigvee x_{3}\left(S x_{3}+x_{1}=x_{2}\right) \equiv\left(x_{1}<x_{2}\right)\right]$.

Definition 7.1. The function $i: S_{A} \rightarrow S_{0}$, is defined, as follows:

(1) $i\left(t_{n}=t_{m}\right)=p_{k}\left(p_{k} \in A t_{0}\right)$

(2) $i\left(t_{n}<t_{m}\right)=p_{s}\left(p_{s} \in A t_{0}\right)$

(3) $i(\sim \phi)=\sim i(\phi)$

(4) $i(\phi F \psi)=i(\phi) F i(\psi)$, for $F \in\{\rightarrow, \vee, \wedge, \equiv\}$

(5) $i\left(\wedge x_{k} \phi\right)=i\left(\bigvee x_{n} \phi\right)=i(\phi)$,

where $\phi, \psi \in S_{A}$.

Definition 7.2. $\left\langle R_{0+}^{P}, L_{2}^{r} \cup X_{r}\right\rangle$ is the Arithmetic System, where $X_{r}=X_{P} \cup Y_{P}$ (see [3], [4], [8]).

In [8], one can read that the System $\left\langle R_{0+}^{P}, L_{2}^{r} \cup X_{r}\right\rangle$ is a modification of Peano's Arithmetic System.

Next,

Definition 7.3. $L_{D}^{r}=\left\{\phi \in L_{2}^{r}: i(\phi) \in T_{D}\right\}$.

\section{Theorem 7.4.}

$\operatorname{Cn}\left(R_{0+}^{P}, L_{D}^{r} \cup X_{r}\right)=\operatorname{Cn}\left(R_{0+}^{P}, L_{2}^{r} \cup X_{r}\right)$, where $X_{r}=X_{P} \cup Y_{P}$.

Proof. Let

(1) $\alpha \in L_{2}^{r}-L_{D}^{r}$

and

(2) $X_{r}=X_{P} \cup Y_{P}$.

From Definition 5.1., Definition 5.5., Definition 7.1. and Definition 7.3., it follows that

(3) $\left(\forall \phi \in L_{2}^{r}\right)\left[\operatorname{Pr}(\phi) \subseteq\{=\} \Rightarrow \phi \in L_{D}^{r}\right]$ and

(4) $\left(\forall \phi \in L_{2}^{r}\right)\left[\operatorname{Pr}(\phi) \subseteq\{<\} \Rightarrow \phi \in L_{D}^{r}\right]$.

From (1), (3) and (4), it follows that

(5) $<\in \operatorname{Pr}(\alpha)$

and

(6) $=\in \operatorname{Pr}(\alpha)$. 
From (1), (3), (4), (5), (6), Definition 5.1.,

Definition 5.5., Definition 7.1., and the definition of the formula $\psi^{12}$, it follows that

(7) $i\left(\psi^{12} \rightarrow \alpha\right) \in T_{D}$

and

(8) $\psi^{12} \rightarrow \alpha \in L_{2}^{r}$.

Hence, from (1), (5), (6), by Definition 5.1.,

Definition 5.5. and Definition 7.3., it follows that

(9) $\psi^{12} \rightarrow \alpha \in L_{D}^{r}$.

Hence, from (1), (2), (3), (4), Definition 7.2. and

Definition 7.3., it follows that

(10) $\operatorname{Cn}\left(R_{0+}^{P}, L_{D}^{r} \cup X_{r}\right)=\operatorname{Cn}\left(R_{0+}^{P}, L_{2}^{r} \cup X_{r}\right)$.

Thus, by the proof of Theorem 7.4., one can obtain (see [17]):

\section{Conclusion 7.5.}

Every Arithmetic System $\left\langle R_{0+}^{P}, L_{2}^{r} \cup X_{r}\right\rangle$ can be based on the system of the Atomic Logic $\left\langle R_{0+}, L_{D}^{r}\right\rangle$, where

$$
\psi^{12} \in \operatorname{Cn}\left(R_{0+}^{P}, L_{2}^{r} \cup X_{r}\right)
$$

or

$$
\begin{aligned}
& \qquad \psi^{14} \in \operatorname{Cn}\left(R_{0+}^{P}, L_{2}^{r} \cup X_{r}\right) \\
& \text { and }\left(\forall \phi \in L_{2}^{r} \cup X_{r}\right)[\operatorname{Pr}(\phi) \subseteq\{=,<\}] \\
& \text { (cf. [1] p.530-541). }
\end{aligned}
$$

\section{References}

[1] B. Buldt. The Scope of Gödel's First Incompleteness Theorem, Logica Universalis, 8:499 - 552, 2014.

[2] Yu. L. Ershov and E. A. Palyutin. Mathematical Logic. Mir Publishers, Moscow, 1984.

[3] A. Grzegorczyk. An Outline of Mathematical Logic.
Fundamental Results and Notions Explained with All Details, D. Reidel Publishing Company, Dordrecht-Holland/Boston-USA, PWN, Warszawa, 1974.

[4] R. Murawski. Recursive Functions And Metamathematics. Problems of Completeness and Decidability, Gödel's Theorems. Springer Science+Business Media Dordrecht, 1999.

[5] W. A. Pogorzelski. The Classical Propositional Calculus. PWN, Warszawa, 1975.

[6] W. A. Pogorzelski. The Classical Calculus of Quantifiers. PWN, Warszawa, 1981.

[7] W. A. Pogorzelski and T. Prucnal. The substitution rule for predicate letters in the first-order predicate calculus. Reports on Mathematical Logic, 5:77 - 90,1975.

[8] H. Rasiowa. Introduction to Modern Mathematics. North-Holland Publishing Company, 1973.

[9] B. Sobocinski. Axiomatization of partial system of three-valued calculus of propositions. The Journal of Computing Systems, 1:23 - 55, 1952.

[10] T. Stepien. System $\overline{\boldsymbol{S}}$. Reports on Mathematical Logic, 15:59-65, 1983.

[11] T. Stepien. System $\overline{\boldsymbol{S}}$. Zentralblatt für Mathematik, 471, 1983.

[12] T. Stepien. Logic based on atomic entailment. Bulletin Of The Section Of Logic, 14:65-71, 1985.

[13] T. Stepien. Logic Based On Atomic Entailment And Paraconsistency. $11^{\text {th }}$ International Congress Of Logic, Methodology And Philosophy Of Science (August 1999, Krakow, Poland).

[14] T. J. Stepien and L. T. Stepien. Atomic Entailment and Classical Entailment, The Bulletin of Symbolic Logic, 17:317 - 318, 2011.

[15] T. J. Stepien and L. T. Stepien. Atomic Entailment and Atomic Inconsistency, $6^{\text {th }}$ International Conference "Non-classical logics. Theory \& Applications" (4 - 6 September 2013, Lodz, Poland).

[16] T. J. Stepien and L. T. Stepien. Atomic Entailment and Atomic Inconsistency and Classical Entailment. Journal of Mathematics and System Science, 5:60 - 71, 2015.

[17] T. J. Stepien and L. T. Stepien. The formalization of the arithmetic system on the ground of atomic logic. (Logic Colloquium 2015, 3 - 8 August 2015, Helsinki, Finland), to appear in The Bulletin of Symbolic Logic. 